\title{
Simultaneous radio and $X$-ray observations of the low-mass X-ray binary GX 13+1
}

\author{
J. Homan ${ }^{1,6}$, R. Wijnands ${ }^{2,4}$, M. P. Rupen ${ }^{3}$, R. Fender ${ }^{4}$, R. M. Hjellming ${ }^{5}$, T. di Salvo ${ }^{4}$, and M. van der Klis ${ }^{4}$ \\ 1 INAF - Osservatorio Astronomico di Brera, via E. Bianchi 46, 23807 Merate, Italy \\ 2 School of Physics and Astronomy, University of St Andrews, St Andrews, Fife KY16 9SS, Scotland, UK \\ 3 National Radio Astronomy Observatory, Socorro, NM 87801, Mexico \\ 4 Astronomical Institute “Anton Pannekoek”, University of Amsterdam, Kruislaan 403, 1098 SJ, Amsterdam, The Netherlands \\ ${ }^{5}$ Deceased \\ ${ }^{6}$ Center for Space Research, MIT, 70 Vassar Street, Cambridge, MA 02139, USA
}

Received 2 September 2003 / Accepted 13 January 2004

\begin{abstract}
We present the results of two simultaneous X-ray/radio observations of the low-mass X-ray binary GX 13+1, performed in July/ August 1999 with the Rossi X-ray Timing Explorer and the Very Large Array. In X-rays the source was observed in two distinct spectral states; a soft state, which had a corresponding $6 \mathrm{~cm}$ flux density of $\sim 0.25 \mathrm{mJy}$, and a hard state, which was much brighter at 1.3-7.2 mJy. For the radio bright observation we measured a delay between changes in the X-ray spectral hardness and the radio brightness of $\sim 40 \mathrm{~min}$, similar to what has been found in the micro-quasar GRS 1915+105. We compare our results with those of GRS 1915+105 and the atoll/Z-type neutron star X-ray binaries. Although it has some properties that do not match with either atoll or Z sources, GX 13+1 seems more similar to the Z sources.
\end{abstract}

Key words. accretion, accretion disks - stars: individual: GX 13+1 - stars: neutron - ISM: jets and outflows X-rays: binaries - radio continuum: stars

\section{Introduction}

Based on their correlated spectral and variability properties, the brightest persistent neutron star low-mass X-ray binaries (LMXBs) are often divided in two groups: the atoll and Z sources (Hasinger \& van der Klis 1989; van der Klis 1995a), after the tracks they trace out in X-ray colour-colour diagrams (CDs). Although atoll and $\mathrm{Z}$ sources share some variability and spectral properties (in the X-ray band), there are significant differences between the two groups: atoll sources are less luminous, have harder X-ray spectra and show stronger rapid time variability than the $\mathrm{Z}$ sources (van der Klis 1995a). Atoll sources are also less luminous in the radio (Fender \& Hendry 2000). Although some of the observational differences can be accounted for by differences in the mass accretion rate, with the $\mathrm{Z}$ sources probably accreting near the Eddington rate and atoll sources at rates ranging from near Eddington to less than $10 \%$ of it, it is generally believed that additional differences, e.g. in the neutron star properties, are required to explain all observational differences (Hasinger \& van der Klis 1989).

The nature of GX $13+1$, one of the brightest neutron star LMXBs, is still ambiguous. Schulz et al. (1989) grouped it with the high luminosity sources (Schulz et al. 1989), which included the six sources that were later labeled as $\mathrm{Z}$ sources.

Send offprint requests to: J. Homan,

e-mail: jeroen@space.mit.edu
Hasinger \& van der Klis (1989) classified it as a bright atoll source, although they noted that of all atoll sources it showed properties which were closest to those seen in the $\mathrm{Z}$ sources, most notably its flaring branch-like appearance in the CD and the featureless power law noise in the power spectrum that is typical for that spectral state. Although the EXOSAT observations analyzed by Hasinger \& van der Klis (1989) only showed the source in the so-called "banana branch" state, the bimodal behavior of the source reported by Stella et al. (1985) strongly suggests that the source occasionally enters a different state. The latter seemed to be confirmed by the first observations of the source with the Rossi X-ray Timing Explorer (RXTE), in which a clear two branched structure was found in the CD (Homan et al. 1998). Although the pattern in the $C D$ resembled both $Z$ and atoll source tracks, the resemblance to $\mathrm{Z}$ sources was strengthened by the discovery (in the same observations) of a 57-69 Hz quasi-periodic oscillation (QPO), which had properties similar to that of the horizontal branch QPO in the Z sources. The CD of GX $13+1$ in a recent paper by Muno et al. (2002), which for the first time displays a "complete" pattern, shows a sharp vertex, similar to the normal branch/flaring branch vertex seen in the $\mathrm{Z}$ sources and quite unlike the rather smooth curves seen in atoll sources. More recently, Schnerr et al. (2003) analyzed a large set of RXTE data and conclude that many of the source's properties do not fit within the atoll/Z framework, although they favor the option of 
an atoll source. They suggest that part of its unusual behavior can be explained with the presence of a relativistic jet, that is almost pointing directly towards us.

Observations in the infrared suggest the presence of a K giant secondary (Garcia et al. 1992; Bandyopadhyay et al. 1999) and a possible orbital or precessional modulation with a period of $\sim 20$ days (Bandyopadhyay et al. 2002); these are properties that are thought to be more typical for $\mathrm{Z}$ sources than for atoll sources. GX 13+1 also shares a common mean radio luminosity (Grindlay \& Seaquist 1986; Garcia et al. 1988) with the $\mathrm{Z}$ sources and black hole candidates (Fender \& Hendry 2000 ), suggesting a similar origin for the quiescent radio emission from persistent black hole and $\mathrm{Z}$ source $\mathrm{X}$-ray binaries, and GX 13+1. None of the (other) atoll sources is consistent with this relation. Although the source is variable in radio on time scales of less than an hour, no clear relation between X-ray and radio luminosities was found (Garcia et al. 1988).

In this paper we present the results of a coordinated $\mathrm{X}$-ray/radio campaign, which had as its principal aim to investigate the possibility that GX $13+1$ shows a similar X-ray state dependence of its radio emission as is found in the $\mathrm{Z}$ sources. In most of those sources the radio luminosity decreases from the horizontal branch to the flaring branch. We find similar behavior in GX $13+1$.

\section{Observations and analysis}

\subsection{X-ray observations}

Our X-ray data were obtained simultaneously with the Proportional Counter Array (PCA; Zhang et al. 1993; Jahoda et al. 1996) and the High Energy X-ray Timing Experiment (HEXTE; Gruber et al. 1996; Rothschild et al. 1998) onboard RXTE (Bradt et al. 1993). The observations were performed in 1999 between July 31 23:52 UTC and August 1 10:39 UTC (obs. 1), and on August 04 between 02:44 and 12:05 UTC (obs. 2). The total exposure times for the two observations were, respectively, $\sim 22.5 \mathrm{ks}$ and $\sim 18.3 \mathrm{ks}$. The PCA and HEXTE data were obtained in several different modes; the spectral and timing properties of these modes are given in Table 1. For all modes we discarded data taken during Earth occultations and passages through the South Atlantic Anomaly.

The Standard 2 data were used to produce light curves, colour curves, a colour-colour diagram (CD), and a hardnessintensity diagram (HID), and to perform a spectral analysis. Only data from Proportional Counter Units (PCUs) 0 and 2 were used (all layers), since these were the only two that were active during all our observations. The data were background subtracted; dead time corrections ( $\sim 3-3.5 \%)$ were only applied for the spectral analysis. A soft colour was defined as the ratio of count rates in the 4.2-7.5 keV and $2.5-4.2 \mathrm{keV}$ energy bands, and a hard colour as the ratio of count rates in the $10.0-18.5 \mathrm{keV}$ and $7.5-10.0 \mathrm{keV}$ energy bands (these four energy bands correspond, respectively, to Standard 2 channels (running from 1 to 129) 7-14, 3-6, 21-40, and 15-20). A CD and a HID were produced by, respectively, plotting hard colour versus soft colour and hard colour versus the $2.5-18.5 \mathrm{keV}$ count rate, for each $16 \mathrm{~s}$ data point. The soft and hard colour
Table 1. Data modes for the RXTE/PCA and RXTE/HEXTE.

\begin{tabular}{lccc}
\hline \hline Mode & $\begin{array}{c}\text { Time } \\
\text { res. (s) }\end{array}$ & $\begin{array}{c}\text { Energy } \\
\text { range }(\mathrm{keV})\end{array}$ & $\begin{array}{c}\text { Energy } \\
\text { channels }\end{array}$ \\
\hline Standard 1 & $1 / 8$ & $2-60$ & 1 \\
Standard 2 & 16 & $2-60$ & 129 \\
SB_125us_0_13_1s & $1 / 8192$ & $2-5.9$ & 1 \\
SB_125us_14_17_1s & $1 / 8192$ & $5.9-7.6$ & 1 \\
E_125us_64M_18_1s & $1 / 8192$ & $7.6-60$ & 64 \\
\hline E_8us_256_DX1F & $1 / 131072$ & $10-250$ & 256 \\
\hline
\end{tabular}

curves, which had an initial time resolution of $16 \mathrm{~s}$, were rebinned to a time resolution of $1024 \mathrm{~s}$, to allow a better study of their variations on long time scales. The PCA spectra were created using the standard FTOOLS V5.2 routines. Systematic errors of $0.6 \%$ were added and response matrices were produced using PCARSP (V8.0). The details of the models used to fit the spectra of GX $13+1$ are discussed in Sect. 3.1.

HEXTE light curves were produced by running the FTOOLS V5.2 script hxtlcurv (which automatically corrects for background and deadtime) on the E_8us_256_DX1F mode data of cluster A. Additional rebinning was applied, resulting in $1024 \mathrm{~s}$ time bins, to achieve better statistics.

The rapid X-ray time variability of the source was studied in terms of power spectra. For this analysis we performed fast fourier transforms (FFTs) of the high time resolution (1/8192 s) data. Power spectra were produced with frequencies of $1 / 1024$ $512 \mathrm{~Hz}(2-26.5 \mathrm{keV}$ band) and $1 / 16-2048 \mathrm{~Hz}(2-26.5 \mathrm{keV}$, 2-6.3 keV, and 6.3-26.5 keV bands). No background or dead time corrections were applied to the data prior to the FFTs; the effects of dead time were accounted for by our fitting method. The power spectra were selected on time, count rate and/or colour and subsequently rms normalized according to a procedure described in van der Klis (1995b). The resulting power spectra were fitted with a constant, to account for the dead-time modified Poisson level, a power law, a zero-centered Lorentzian, to account for the deviations from the power law noise, and a Lorentzian for a weak QPO in the second observation. Errors on the fit parameters were determined using $\Delta \chi^{2}=1$ (67\% confidence) for a single parameter. Upper limits on QPOs were determined by fixing the frequency and $F H W M$ to (a range of) values and using $\Delta \chi^{2}=2.71$ (95\% confidence).

\subsection{Radio observations}

The radio data were obtained with the Very Large Array (VLA) radio observatory in its most extended (A) configuration. GX 13+1 was observed at $6 \mathrm{~cm}$ on 1999 August 1 between 00:33 and 09:31 UTC (obs. 1), and on 1999 August 4 between 00:22 and 08:51 UTC (obs. 2). During the first observation 25 of the 27 VLA antennas were used; 26 were used during the second observation. Due to technical problems $\sim 39 \%$ (obs. 1) and $\sim 8 \%$ (obs. 2) of the total observing time was lost. Observations were made simultaneously in both circular polarizations in each of two independent $50 \mathrm{MHz}$ bands centered on 4885.1 and 4835.1 MHz. Flux densities, which are 
all Stokes $I$, were referenced to those of $1328+307$ (3C 286), taken to be 7.462 and $7.510 \mathrm{Jy}$ at 4885.1 and $4835.1 \mathrm{MHz}$ respectively (Perley et al., priv. comm.). The overall flux density scale is probably good to at least $\sim 5 \%$. To calibrate the complex antenna gains the standard calibration source 1817-254 was observed at $30 \mathrm{~min}$ (obs. 1) or $6 \mathrm{~min}$ (obs. 2) intervals; the reason for the denser sampling of the calibration source in obs. 2 was that during obs. 1 a large part of the data was rendered useless due to a combination of bad weather conditions and too long intervals between calibration observations. The data were reduced and analyzed using the Astronomical Image Processing System (AIPS). For each day, images were first made using the entire data sets, ignoring any variability of GX $13+1$. These images were used to find nearby confusing sources, which were then subtracted from the original uv-data. These uv-data were then split into 15-min bins (or longer, in the case of weak signals) and imaged to give the flux density history of GX $13+1$. This procedure allows the full synthesis mapping of confusing sources, while retaining the best possible sensitivity to fluctuations of GX 13+1. The flux densities are the average of the of maximum flux density and the integrated flux density in the region containing the source in the cleaned image. Note that because of calibration and other "dead" time, the amount of data in each bin is usually less than the sampling time.

There are two main sources of error in the radio flux densities: (1) statistical (thermal) noise and (2) calibration errors. We estimated the first by measuring the rms background noise near the source. For sources this faint the main calibration error comes from poor phase transfer from the calibrator to the source, which leads to phase errors which scatter the flux density of the source across the image. This is the equivalent of optical seeing, and the true point spread function is broader than that calculated assuming perfect phasing. For our point source, we estimated the magnitude of this effect by comparing the peak flux density with the integral over the apparent emission; this is roughly equivalent to comparing the original peak to that obtained by convolving the image down to a lower resolution. As a conservative estimate we take the rms calibration error to be the difference between the peak and the integrated flux density. This was then added in quadrature to the off-source rms noise to give the error bars used in Fig. 1j. The effects of these calibration errors can be seen in the increase of the error bars early in obs. 1 and during the beginning and end of obs. 2; this is mainly due to a combination of poor weather and large differences in elevation between the calibrator and the source at these times.

\section{Results}

\subsection{X-ray observations}

From Figs. 1-3 it is clear that the X-ray properties of the source changed between the first to the second observation. The second observation has a higher count rate, both in the 2-25 keV (Figs. 1a and b) and 20-100 keV bands (Figs. 1c and d) and shows more variability in the $2-25 \mathrm{keV}$ band. Also, the second observation tends to be spectrally harder; while the PCA count rates increased only by a factor of $\sim 1.3$, the HEXTE count rates increased by a factor of $\sim 3.2$. The spectral difference is most clearly seen in the CD and HID shown in Fig. 2, where the two observations show up as distinct patches, and Fig. 3, which shows the ratio of the spectra of obs. 2 and 1 . Note that the small bridge between the two patches in the HID (Fig. 2b) does not represent a real connection between the two patches, but corresponds to the upper right part of patch traced out during obs. 1 in the CD. It is not clear from our observations how the source bridges the gap between the two patches in HID we refer to Schnerr et al. (2003) for an observation that shows a transition between the two patches. Although type I X-ray bursts have been observed from GX 13+1 (Fleischman 1985; Matsuba et al. 1995), none were seen during our observations.

\subsubsection{X-ray spectra}

X-ray spectral fits were performed with XSPEC (Arnaud 1996,V11.2) to the $3-25 \mathrm{keV}$ PCA spectra using two different models. The first model is based on the continuum model used by Ueda et al. (2001), for their ASCA 1-10 keV spectra of GX 13+1, and Sidoli et al. (2002), for their 2-10 keV XMM-Newton spectra. It consists of a black body (bbody in XSPEC), and a disk black body (diskbb). Galactic neutral hydrogen absorption (phabs) was fixed at $N_{\mathrm{H}}=2.9 \times$ $10^{22}$ atoms cm$~^{-2}$ (Ueda et al. 2001). A reasonable fit was found for obs. $2\left(\chi_{\text {red }}^{2}=1.52\right.$ - with an unphysically small disk radius), but not for obs. $1\left(\chi_{\text {red }}^{2}=11.3\right)$. Adding a line (gauss) around $6.4 \mathrm{keV}$ and an absorption edge (edge) around $8 \mathrm{keV}$ (Ueda et al. 2001) did not lead to acceptable fits. The second model we tried was a thermal Comptonization model (comptt, Titarchuk 1994; Hua \& Titarchuk 1995), which has recently been applied successfully to the broad band continuum of several bright neutron star LMXBs (see, e.g., di Salvo et al. 2000; Iaria et al. 2001). The $N_{\mathrm{H}}$ was again fixed to $N_{\mathrm{H}}=2.9 \times$ $10^{22}$ atoms $\mathrm{cm}^{-2}$. For the second observation a reasonable fit was obtained $\left(\chi_{\text {red }}^{2}=1.35\right)$, but the first observation showed large residuals between 5 and $10 \mathrm{keV}\left(\chi_{\text {red }}^{2}=15.6\right)$. Adding a line around $6.4 \mathrm{keV}$ and an edge around $9 \mathrm{keV}$ greatly improved the fit $\left(\chi_{\text {red }}^{2}=0.6\right)$. For the second observation the addition of the line and edge led to a small improvement $\left(\chi_{\text {red }}^{2}=\right.$ 0.9 ), with the two components not being detected significantly; although the improvement of the fit was statistically significant the normalizations of the two components were consistent with zero. The best-fit results with the four-component model are given in Table 2. Some caution should be taken with interpreting the spectral results, as the low line energy (which tended to decrease when not fixed) and the high edge energy indicate different ionization stages of Fe. Also the large spectral changes within obs. 1 may have been partly responsible for the observed residuals. For both observations no black body component was needed, as the Comptonization component provided good fits at low energies.

For a more model independent comparison of the spectra of the two observations we plot the ratio of obs. 2 and 1 in Fig. 3. While below $7 \mathrm{keV}$ the ratio is rather constant, above that energy the spectrum of obs. 2 becomes increasingly hard. 

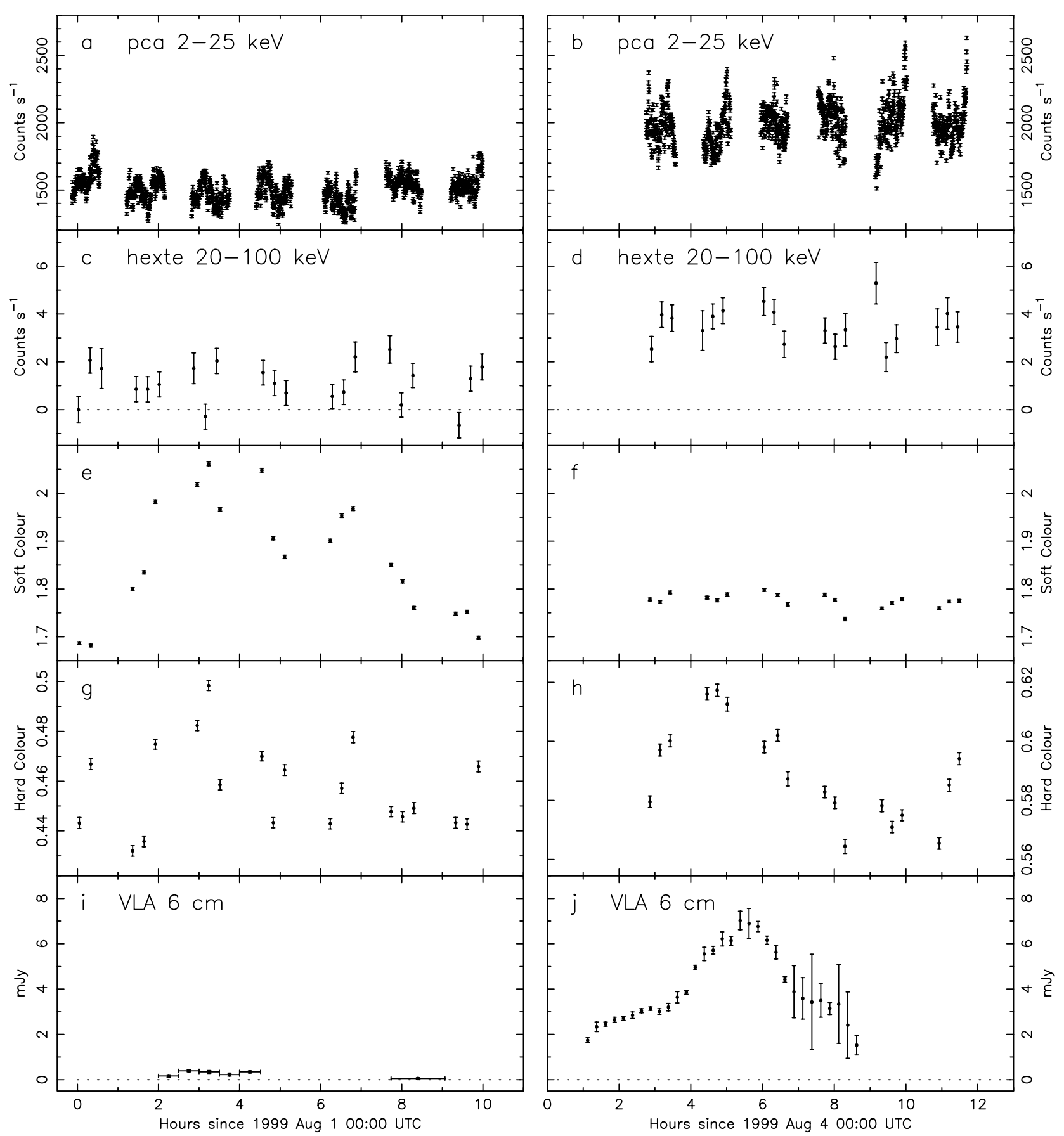

Fig. 1. The evolution of GX 13+1 during the observations on 1999 August 1 (left column) and 1999 August 4 (right column). a), b): PCA Count rate in the 2-25 keV range (16 s bins). c), d): HEXTE 20-100 keV count rate (rebinned to $1024 \mathrm{~s}$ ). e), f): Soft color (rebinned to $1024 \mathrm{~s}$ ). g), h): Hard color (rebinned to $1024 \mathrm{~s}$ ). i), j): VLA $6 \mathrm{~cm}$ radio flux density (1800-4800 s [i] and $900 \mathrm{~s}$ [j]). Errors on the flux density in panel i are of the order of $50 \mu \mathrm{Jy}$. For the PCA count rate and colours only data from PCUs 0 and 2 were used. For the HEXTE count rate only Cluster A was used. Note that all quantities have the same ranges for obs. 1 and 2, except for the hard colour (to allow an easier comparison with changes in the radio flux density).

\subsubsection{X-ray variability}

From the PCA light curves in Fig. 1 it is clear that, at least on time scales of minutes to hours, the source was more variable during the second observation. The $2-26.5 \mathrm{keV}$ power spectra of the two observations are shown in Fig. 4; the results of the power spectral fits are given in Table 3. Both power spectra are relatively featureless and their continuum can be well fit by a combination of power law noise and a zero-centered Lorentzian. While the power law noise has an identical index in the two observations, the FHWM of the two zero-centered 


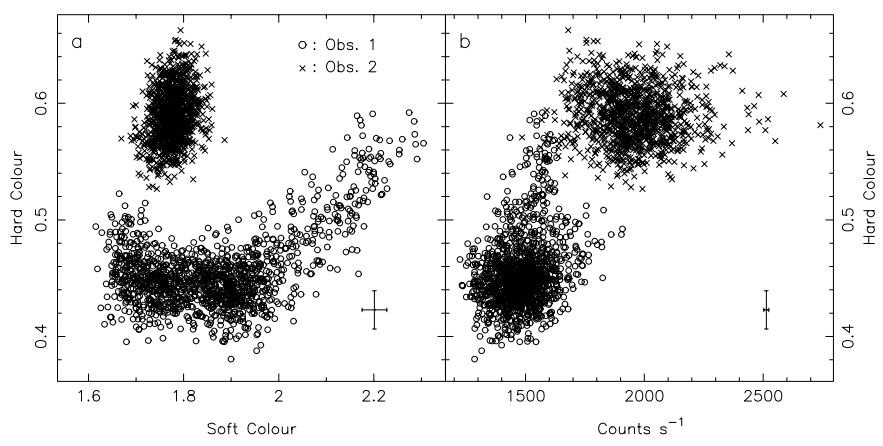

Fig. 2. a) Colour-colour diagram and b) hardness-intensity diagram for the two RXTE/PCA observations of GX 13+1. Points of the first observations are depicted by the open circles, those of the second observation by crosses. Each data point represent a $16 \mathrm{~s}$ interval. Typical error bars are shown in the lower-right corners.

Lorentzians, and hence their maxima in a $v P_{v}$ plot, differ by more than two orders of magnitude, suggesting that they might not be related. We added a Lorentzian to the fit function of the second observation, for a QPO at $60 \mathrm{~Hz}$ - this QPO has a statistical significance of $3.2 \sigma$. No similar QPO was found in the first observation. We also measured the total rms in two frequency intervals; $0.001-1 \mathrm{~Hz}$ and $1-100 \mathrm{~Hz}$. In both frequency intervals the fractional variability is stronger in the second observations, with the difference being more pronounced at high frequencies. We searched for high frequency QPOs in the 1/16-2048 Hz power spectra with the $F W H M$ fixed to $150 \mathrm{~Hz}$, but only upper limits could be determined. For obs. 1 they were: $2.1 \%(2-26.5 \mathrm{keV}), 2.8 \%(2-6.3 \mathrm{keV})$, and $4.0 \%$ $(6.3-26.5 \mathrm{keV})$ and those for obs. $2: 2.2 \%(2-26.5 \mathrm{keV}), 3.7 \%$ (2-6.3 keV), and 3.6\% (6.3-26.5 keV).

\subsection{Radio observations}

During the first observation the source was only sometimes detected significantly, at a very low level and with low variability; the average flux density was $\sim 0.25 \mathrm{mJy}$. The radio flux density in the second observations was much higher, varying between 1.3 and $7.2 \mathrm{mJy}$. The flux density showed a slow increase for about $4.5 \mathrm{~h}$, followed by a gradual decrease. No significant variability was detected on time scales less than $15 \mathrm{~min}$. An upper limit on the size of the source of 0.15 arcsec was obtained.

\subsection{X-ray/radio comparison}

Comparing panels $i$ and $j$ of Fig. 1 with panels $a$ and $b$ of the same figure shows that, apart from the fact that the second observation is brighter both in X-rays and radio, there is no apparent relation between X-ray count rate and radio flux, at least not on the time scales on which the latter varies. On the other hand, a comparison of the radio flux and hard X-ray colour of obs. 2 reveals similar broad peaks centered around 205:00 UTC. As is obvious from panels $1 \mathrm{~h}$ and $1 \mathrm{j}$ there exists a significant lag between the peaks of the hard colour curve and the radio light curve. Fitting the broad peaks in the hard color (02:52-06:02 UTC) and radio curves (03:53-06:53 UTC) both with a Lorentzian gives a lag between the two peaks of $42 \pm$ 3 min.

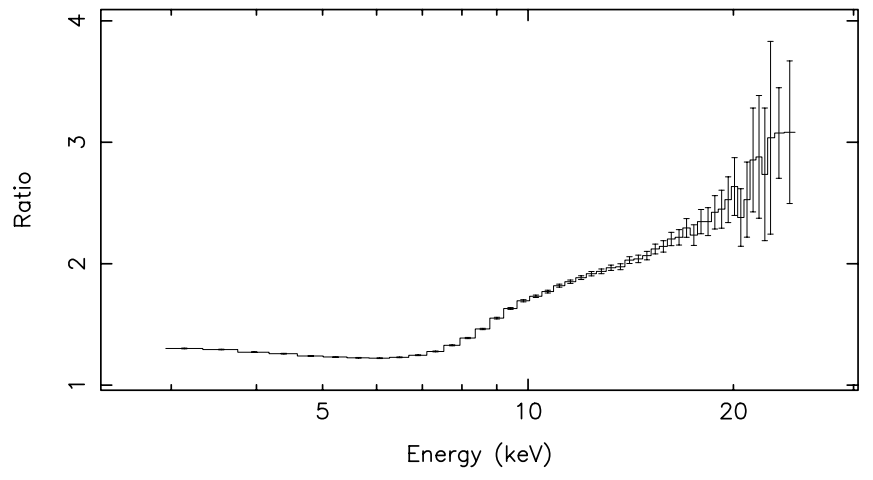

Fig. 3. The ratio of the mean raw RXTE count rate spectrum of obs. 2 to that of obs. 1, revealing an overall increase and a relative hardening of the spectrum.

Table 2. Spectral fit results. Errors on the fit parameters are $68 \%$ confidence limits, upper limits represent $95 \%$ confidence limits. The 3-10 and $10-25 \mathrm{keV}$ fluxes are unabsorbed.

\begin{tabular}{|c|c|c|}
\hline Parameter & Obs. 1 & Obs. 2 \\
\hline$N_{\mathrm{H}}\left(\right.$ atoms $\left.\mathrm{cm}^{-2}\right)$ & $2.910^{22}$ (fixed) & $2.910^{22}$ (fixed) \\
\hline$k T_{W}(\mathrm{keV})$ & $0.98 \pm 0.02$ & $0.92 \pm 0.01$ \\
\hline$k T_{\mathrm{e}}(\mathrm{keV})$ & $2.88 \pm 0.06$ & $3.04 \pm 0.04$ \\
\hline$\tau$ & $7.2 \pm 0.2$ & $8.10 \pm 0.15$ \\
\hline$R_{W}(\mathrm{~km})$ & $21.7 \pm 0.9$ & $27.2 \pm 0.7$ \\
\hline$E_{\mathrm{FE}}(\mathrm{keV})$ & 6.42 (fixed) & 6.42 (fixed) \\
\hline$F W H M(\mathrm{keV})$ & $0.78 \pm 0.13$ & $0.4_{-0.4}^{+0.2}$ \\
\hline $\mathrm{Fe} E W(\mathrm{eV})$ & 223 & $<96$ \\
\hline$E_{\text {edge }}(\mathrm{keV})$ & $9.1 \pm 0.1$ & 9.1 (fixed) \\
\hline$\tau_{\text {edge }}$ & $0.12 \pm 0.03$ & $<0.03$ \\
\hline$F_{3-10}\left(\operatorname{ergs~cm}{ }^{-2} \mathrm{~s}^{-1}\right)$ & $1.5 \times 10^{-8}$ & $2.0 \times 10^{-8}$ \\
\hline$F_{10-25}\left(\mathrm{ergs} \mathrm{cm}^{-2} \mathrm{~s}^{-1}\right)$ & $1.8 \times 10^{-9}$ & $3.6 \times 10^{-9}$ \\
\hline$\chi_{\text {red }}^{2}$ (d.o.f.) & $0.6(40)$ & $0.9(41)$ \\
\hline
\end{tabular}

Fit parameters of the comptt model $-k T_{W}$ : input soft photon (Wien) temperature $-k T_{\mathrm{e}}$ : plasma temperature $-\tau$ : plasma optical depth $R_{W}$ : effective Wien radius for the soft seed photons (in 't Zand et al. 1999), here derived for an assumed distance of $7 \mathrm{kpc}$.

\section{Discussion}

We have observed the neutron star LMXB GX 13+1 simultaneously in X-rays and radio. The source was found in two clearly distinct X-ray states; the spectrally hard state had associated radio fluxes that were between 4 and 18 times higher than the maximum we detected in the softer X-ray state. This dependence of the radio flux on X-ray state is similar to what is found for other bright neutron star LMXBs (the Z sources; see Hjellming \& Han 1995, and references therein) and more recently also in the less-luminous atoll source 4U 1728-34 (Migliari et al. 2003). More specifically, our observations strongly suggest that the radio flux of the source is related to the X-ray spectral hardness, on time scales of both hours and days. The fact that the short term correlation was only observed 


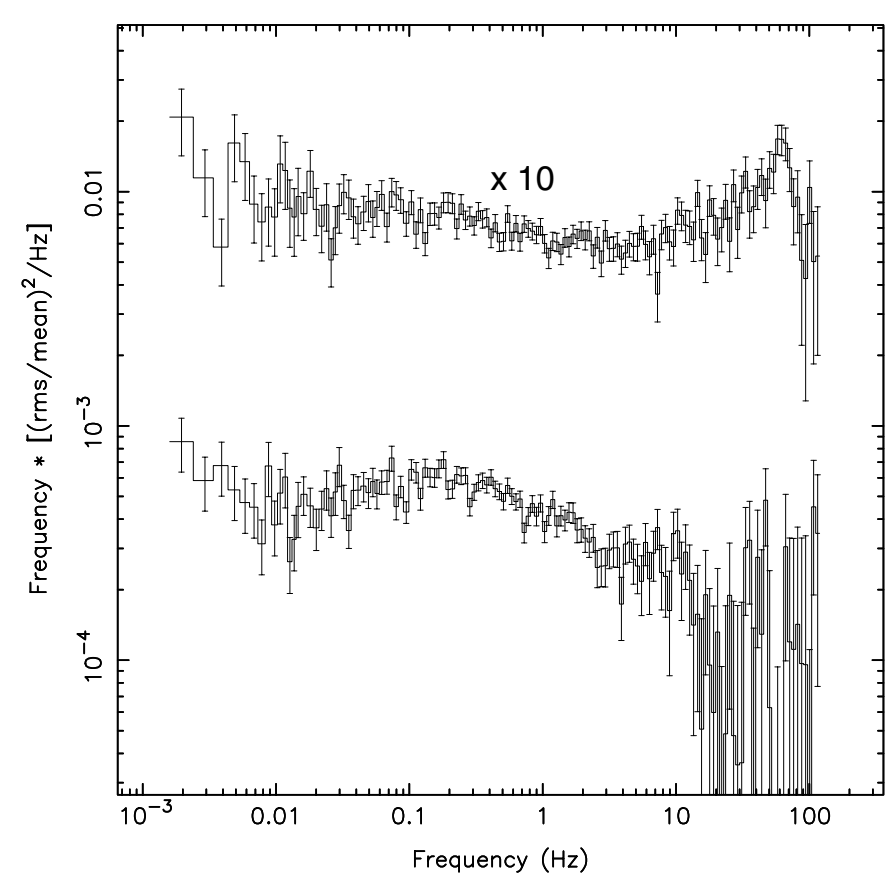

Fig. 4. Power spectra $(2-26.5 \mathrm{keV})$ of the two RXTE/PCA observations (lower: obs. 1, upper: obs. 2) in an $v P(v)$ representation. Note that the upper power spectrum has been multiplied by a factor of 10 , to avoid overlapping of the power spectra. The Poisson level has in both cases been subtracted for plotting purposes.

in X-ray hardness and not in X-ray count rate can be understood from Fig. $2 b$, which shows that the $2-25 \mathrm{keV}$ count rate and hard color are not correlated at all during obs. 2 . It should also be noted that there was only one broad radio flare in our observations, making it hard to judge the true significance of the observed lag. Future observations are planned to search for additional evidence that supports/challenges the observed lag. During the remainder of the discussion we assume that the broad features in the radio and hardness curves are related and that the lag is therefore significant.

\subsection{Outflow}

It is generally believed that the radio emission from neutron star and black hole X-ray binaries is produced by highly relativistic electrons that interact with magnetic fields to produce synchrotron radiation. High resolution radio observations of a handful of X-ray binaries show that these electrons reside in powerful, collimated outflows, commonly referred to as jets. It is assumed that in the X-ray binaries for which jets have not (yet) been directly observed the radio emission originates in a similar outflow. To see whether this could also be the case for GX $13+1$, we estimate the size of the emission region. If, for an assumed spherical region, this size is larger than the binary separation, the highly relativistic synchrotron plasma cannot be contained in the system and the most plausible option would then be that we are dealing with an outflow. Assuming a maximum brightness temperature $T_{\mathrm{B}}$ of $\leq 10^{12} \mathrm{~K}$ (see, e.g., Kellermann \& Pauliny-Toth 1969) and a distance of $7 \mathrm{kpc}$ (Bandyopadhyay et al. 1999) we derive,
Table 3. Power spectral fit results. As a model independent measure of the variability we also include the total fractional rms in the $0.001-1 \mathrm{~Hz}$ and $1-100 \mathrm{~Hz}$ ranges. FWHM stands for full-width-at-half-maximum.

\begin{tabular}{lcc}
\hline \hline Parameter & Obs. 1 & Obs. 2 \\
\hline Power law rms $^{a}(\%)$ & $5.15 \pm 0.12$ & $7.71 \pm 0.11$ \\
Power law index & $1.09 \pm 0.01$ & $1.09 \pm 0.01$ \\
Lorentzian rms $^{b}(\%)$ & $2.99 \pm 0.01$ & $3.4 \pm 0.6$ \\
Lorentzian FWHM (\%) & $0.45 \pm 0.04$ & $100 \pm 30$ \\
Lorentzian freq. (Hz) & $0($ fixed $)$ & $0($ fixed $)$ \\
QPO rms $(\%)$ & $<1$ & $2.1 \pm 0.4(3.2 \sigma)^{d}$ \\
QPO FHWM $(\mathrm{Hz})$ & $18($ fixed $)$ & $18 \pm 6$ \\
QPO frequency $(\mathrm{Hz})$ & $60($ fixed $)$ & $60 \pm 2$ \\
rms [0.001-1 Hz] $(\%)$ & $6.6 \pm 0.02$ & $9.15 \pm 0.02$ \\
rms [1-100 Hz] $(\%)$ & $3.17 \pm 0.10$ & $5.93 \pm 0.06$ \\
$\chi_{\text {red }}^{2}$ (d.o.f.) & $1.07(209)$ & $1.01(210)$ \\
\hline
\end{tabular}

${ }^{a}$ Integrated between 0.001 and $1 \mathrm{~Hz} ;{ }^{b}$ Integrated between 0 and $+\infty \mathrm{Hz} ;{ }^{c}$ Integrated between $-\infty$ and $+\infty \mathrm{Hz} ;{ }^{d}$ Significance is calculated from the power, not the fractional rms.

following Fender (2003), a minimum size for the emitting region of $\sim 15 R_{\odot}\left(10^{12} \mathrm{~cm}\right)$ at the peak of the radio flare in obs. 2 . A size estimate based on the fastest part $(1.5 \mathrm{~h})$ of the rise of the radio flare $\left(\leq c \Delta t \sim 1.6 \times 10^{14} \mathrm{~cm}\right)$ is consistent with this value. Even for a system with equal masses for the primary and secondary and an orbital period of 20 days the emission region is at least comparable and probably larger than the size of the binary system $\left(\sim 3 \times 10^{12} \mathrm{~cm}\right)$, suggesting an outflow is also present in GX $13+1$.

\subsection{X-ray/radio connection}

Although the exact mechanism for producing jet outflows in $\mathrm{X}$-ray binaries is still not clear, the energy needed to achieve the inferred observed high bulk velocities suggests that they form in the inner parts of the accretion flow, where also most of the $\mathrm{X}$-rays are produced. Simultaneous X-ray and radio observations of GRS $1915+105$ seem to confirm such a direct link between the outflow and inner accretion disk (Mirabel et al. 1998; Klein-Wolt et al. 2002). Clear patterns of X-ray/radio behavior are also observed in other black hole and Z source X-ray binaries; in general the spectrally hard X-ray states have a higher radio luminosity than the spectrally soft states.

Our observations of GX 13+1 reveal a similar pattern, with the second observation being both more luminous in the radio and showing a harder energy spectrum (Figs. 1-3). While the change between the soft and hard spectral states of GX 13+1 most likely occurred on a time scale of one or two days, we also find a relation between the $\mathrm{X}$-ray and radio properties on a time scale of a few hours: a hardening of the X-ray spectrum was followed by an increase in the radio luminosity with a delay of $\sim 40 \mathrm{~min}$. This is the first time such a short-term X-ray/radio connection and delay have been found and measured in a 
neutron star LMXB. It is interesting to note that a similar delay has also been found on several occasions in the galactic black hole X-ray binary GRS $1915+105$ (Klein-Wolt et al. 2002). In that case however, the delay $(\sim 45-60 \mathrm{~min})$ was with respect to the start of the dips in the RXTE/PCA count rate. These dips coincided with a strong spectral hardening. Also, the X-ray dips and radio flares in GRS 1915+105 were a rapid recurring phenomenon, whereas the radio flare in GX $13+1$ was more likely a singular event. Klein-Wolt et al. (2002) explained the observed delay in GRS $1915+105$ as the time it takes for the flow to become optically thin in the radio; this effect is clearly observed in Fig. 9 of Dhawan et al. (2000), which shows light curves of the directly imaged jet of GRS $1915+105$ at different radio wavelengths that peak later with increasing wavelength (see also Pooley \& Fender 1997). Observations of Sco X-1 (Fomalont et al. 2001) suggest that such a delay can also result from the transfer time of the energy from the core to the radio lobes.

The ratio of (radio flux density change)/(X-ray flux change) is much larger in GX $13+1$ than in GRS $1915+105$. If we assume that the inner disk is ejected to form the outflow, as has been proposed for GRS $1915+105$ to explain the X-ray variations, our second observation of GX $13+1$ suggests that a smaller fraction of the inner disk is ejected in this case. The fact that clear dips are observed in GRS 1915+105 might be related to the different nature of the compact object, or to a much larger amount of matter being expelled. Following Fender (2003) we can estimate the mean power of the radio event in obs. 2 to be $\sim 1.8 \times 10^{36} \mathrm{erg} \mathrm{s}^{-1}$. While this is only $\sim 1$ percent of the 3-25 keV X-ray luminosity $\left(\sim 1.4 \times 10^{38} \mathrm{erg} \mathrm{s}^{-1}\right)$, the kinetic energy associated to the (possibly relativistic) outflow may increase this number to a larger fraction of the total accretion energy.

Although short term variations in the radio luminosity of GX $13+1$ have been found before, they were only compared to the X-ray count rates (Garcia et al. 1988), and not to the X-ray spectral properties. No correlations between radio luminosity and X-ray count rate were found, most likely because X-ray data were only available for the radio weak part of their data set. The time scale of shortest radio fluctuations observed by Garcia et al. (1988) (a few hours) is consistent with that of the flare in our second observation. The $6 \mathrm{~cm}$ peak flux density they measured was $2.2 \mathrm{mJy}$, about a factor of $\sim 3.3$ lower than our maximum flux density. Interestingly, when fitting our X-ray spectra with the same $N_{\mathrm{H}}$ used by Garcia et al. (1988) and using their assumed distance of $7 \mathrm{kpc}$ we find a similar difference for the 1-20 keV luminosity.

\subsection{Comparison with $Z$ and atoll source observations}

Before discussing the nature of GX $13+1$ in view of the atoll/Z source classification we compare its X-ray/radio behavior with that of sources from both classes. Although Schnerr et al. (2003) concluded that GX 13+1 is neither a $\mathrm{Z}$ nor an atoll source, one could, purely based on its appearance in the $C D$, argue that the source was in the atoll source banana branch and island state, during obs. 1 and 2 respectively, or the $\mathrm{Z}$ source flaring branch and normal branch.
Table 4. Observed mean and maximum radio flux densities and estimated distances for the six Z sources and GX $13+1$. This table is reproduced from Tables 2 and 3 in Fender \& Hendry (2000), with updated values for GX $13+1$.

\begin{tabular}{lcccc}
\hline \hline Source & $\begin{array}{c}\text { Mean }^{a} \\
(\mathrm{mJy})\end{array}$ & $\begin{array}{c}\text { Max }^{b} \\
(\mathrm{mJy})\end{array}$ & $\begin{array}{c}\text { distance } \\
(\mathrm{kpc})\end{array}$ & Refs \\
\hline Sco X-1 & $10 \pm 3$ & 22 & $2.0 \pm 1.0$ & 1,2 \\
GX 17+2 & $1.0 \pm 0.3$ & 13.4 & $7.5 \pm 2.3$ & 1,3 \\
GX 349+2 & $0.6 \pm 0.3$ & 1.3 & $5.0 \pm 1.5$ & 4,5 \\
Cyg X-2 & $0.6 \pm 0.2$ & 3.4 & $8.0 \pm 2.4$ & $1,6,7$ \\
GX 5-1 & $1.3 \pm 0.3$ & 1.6 & $9.2 \pm 2.7$ & 1 \\
GX 340+0 & $0.6 \pm 0.3$ & 0.6 & $11.0 \pm 3.3$ & 8 \\
GX 13+1 & $1.8 \pm 0.3$ & 7.7 & $7 \pm 1$ & $9-13$ \\
\hline
\end{tabular}

${ }^{a}$ Mean cm radio flux density.

${ }^{b}$ Maximum radio flux density at $6 \mathrm{~cm}$.

Refs. 1: Penninx (1989); 2: Bradshaw et al. (1999); 3: Penninx et al. (1988); 4: Cooke \& Ponman (1991); 5: Christian \& Swank (1997); 6: Hjellming et al. (1990a); 7: Cowley et al. (1979); 8: Penninx et al. (1993); 9: Grindlay \& Seaquist (1986); 10: Garcia et al. (1988); 11: Berendsen et al. (2000); 12: Bandyopadhyay et al. (1999); 13: this work.

Based on observing campaigns of the $\mathrm{Z}$ sources GX 17+2 (Penninx et al. 1988), Cyg X-2 (Hjellming et al. 1990a), Sco X-1 (Hjellming et al. 1990b) and GX 5-1 (Tan et al. 1992), Penninx (1989) suggested that all $Z$ sources share a common radio, UV, and X-ray luminosity on their normal branch. Subsequent detections of GX 349+2 (Cooke \& Ponman 1991) and GX 340+0 (Penninx et al. 1993) at approximately the predicted radio brightness seemed to confirm this idea. Penninx (1989) derived a normal branch luminosity of $\sim 1.6 \times 10^{38} \mathrm{erg} \mathrm{s}^{-1}$ in the $1.5-15 \mathrm{keV}$ band. In the same energy band we obtain (extrapolating our fit to lower energies) $\sim 1.7 \times 10^{38} \mathrm{erg} \mathrm{s}^{-1}$, which is remarkably close the value of Penninx (1989).

The assumption of Penninx (1989) might be not completely valid; using the distance estimates and average flux densities from Fender \& Hendry (2000) (see Table 4) it seems that there is a considerable spread in the average radio luminosity of the six $\mathrm{Z}$ sources. Moreover, none of them has a higher value than GX $13+1$. However, this average radio luminosity depends strongly on the time a source spends in a radio bright state. Taking the maximum radio flux densities (at $6 \mathrm{~cm}$ ) reported in the literature (see references in Table 4) should partly correct for this, which results in GX $17+2$ being the most luminous with GX 13+1 being second. The overall behavior of the radio brightness along the track in the $C D$ is also similar to that observed in several of the Z sources (Penninx et al. 1988; Hjellming et al. 1990a,b).

The only atoll source for which a clear pattern in the $\mathrm{X}$-ray/radio emission has been found is 4U 1728-34 (Migliari et al. 2003). In that source the radio flux density was highest in the island state; it increased by a factor $\sim 6$ from the hard part of the island state toward the transition between the island state and, what seemed to be, the (softer) banana branch. After this transition it dropped sharply by a factor of $\sim 6$. 
While the decrease of radio flux with spectral hardness (in the island state) is opposite to overall behavior in GX $13+1$ and several $\mathrm{Z}$ sources (where we see an increase with spectral hardness along the track in the $\mathrm{CD}$ ), the factor of $\sim 6$ difference between the island state and banana branch is similar in sign and magnitude to the difference between our second and first observation. The average X-ray luminosity of 4U 1728-34 (assuming a distance of $5.2 \mathrm{kpc}$, Galloway et al. 2003) is more than a factor 10 lower than that of GX 13+1, whereas the $6 \mathrm{~cm}$ flux density is probably more than a factor 100 lower.

\subsection{Z or atoll?}

The nature of GX $13+1$ and its place in the Z/atoll classification scheme have recently been extensively studied and discussed by Schnerr et al. (2003). They found that its motion through the HID is in the opposite sense to that in the CD, with the $X$-ray count rate increasing again when the source moves into the spectrally hard state, contrary to most atoll and $\mathrm{Z}$ sources (see our Fig. 2 and also Wijnands et al. (1997) for similar behavior in the $\mathrm{Z}$ source Cyg X-2). Moreover, the strength of the very low frequency variability also changes in the opposite sense to that observed in $\mathrm{Z}$ and atoll sources.

In an attempt to fit GX $13+1$ within the Z/atoll scheme as an atoll source, Schnerr et al. (2003) tried to explain the source's peculiar behavior with the presence of a relativistic jet with an axis nearly aligned to our line of sight. The radio emission could then be boosted by more than an order of magnitude, putting the radio emission of GX $13+1$ in accordance with measurement and upper limits of other atoll sources; the unusual X-ray phenomena could be the consequence of a better view of the X-ray emitting regions associated with the base of the jet, possibly assisted by Doppler boosting.

As we showed above, the X-ray and radio luminosities of GX $13+1$ are actually in the range expected for $\mathrm{Z}$ sources, without requiring any unusual jet geometries, making a $\mathrm{Z}$ source nature more likely in our opinion (the atoll sources GX 9+1, GX 9+9 and GX 3+1 have also similar Xray luminosities but much lower radio luminosities). This is supported by our spectral fit parameters, which are quite similar to those found in the $\mathrm{Z}$ sources (see, e.g., di Salvo et al. 2000, 2001) and less like those in the atoll sources (see, e.g., di Salvo et al. 2000; Barret et al. 2000; Barret \& Olive 2002), and by the fact that GX $13+1$ rarely shows type I X-ray bursts. Based on the morphology in our CD GX 13+1 was probably observed in the normal branch and flaring branch. Although no clear indications for normal branch oscillations are found, the band limited noise measured by Schnerr et al. (2003) has properties similar to the normal branch oscillations found in GX 5-1 (Jonker et al. 2002) and GX 340+0 (Jonker et al. 2000) close to the normal branch/flaring branch vertex, where it is very broad $(Q<1)$ and rather weak ( $\leq 2 \% \mathrm{rms})$. In GX 5-1 and GX $340+0$ this broad bump evolves into a strong $6 \mathrm{~Hz}$ QPO as the spectrum hardens - this is not observed in GX $13+1$. There are additional differences with the other $Z$ sources, in particular with respect to the behavior of the low frequency variability.
Finally, we note that although the source was more similar to the $\mathrm{Z}$ sources than to the atoll sources during our observations (at a luminosity three times as high as during the EXOSAT era), this does not exclude that at lower luminosities it behaves more atoll-like.

\section{Summary}

Our simultaneous X-ray/radio observations of GX $13+1$ revealed a strong dependence of the radio brightness on the $X$-ray state of the source. In the hard spectral state, which was at least 4 times brighter in the radio than the soft state, we also found a correlation between the X-ray and radio properties on a short time scale, with changes in radio having a delay of $\sim 40 \mathrm{~min}$ with respect to those in X-rays. We attribute this delay to the time it takes for the flow to become optically thin in the radio. The absence of strong dips in the X-ray light curve during the radio flare suggests that only a small amount of the matter is redirected from the inflow to the outflow. On the basis of a comparison with atoll and $\mathrm{Z}$ sources we conclude that the source is more similar to the $\mathrm{Z}$ sources, although several properties of GX 13+1 remain unexplained.

Acknowledgements. J.H. thanks Roald Schnerr for comments on an earlier version of the manuscript and Jon Miller for his help with the spectral data reduction. J.H. also acknowledges support from Cofin-2000 grant MM02C71842. The National Radio Astronomy Observatory is a facility of the National Science Foundation operated under cooperative agreement by Associated Universities, Inc.

\section{References}

Arnaud, K. A. 1996, in Astronomical Data Analysis Software and Systems V, 5, ASP Conf. Ser., 101, 17

Bandyopadhyay, R. M., Charles, P. A., Shahbaz, T., \& Wagner, R. M. 2002, ApJ, 570, 793

Bandyopadhyay, R. M., Shahbaz, T., Charles, P. A., \& Naylor, T. 1999, MNRAS, 306, 417

Barret, D., \& Olive, J. 2002, ApJ, 576, 391

Barret, D., Olive, J. F., Boirin, L., et al. 2000, ApJ, 533, 329

Berendsen, S. G. H., Fender, R., Kuulkers, E., Heise, J., \& van der Klis, M. 2000, MNRAS, 318, 599

Bradshaw, C. F., Fomalont, E. B., \& Geldzahler, B. J. 1999, ApJ, 512, L121

Bradt, H. V., Rothschild, R. E., \& Swank, J. H. 1993, A\&AS, 97, 355

Christian, D. J., \& Swank, J. H. 1997, ApJS, 109, 177

Cooke, B. A., \& Ponman, T. J. 1991, A\&A, 244, 358

Cowley, A. P., Crampton, D., \& Hutchings, J. B. 1979, ApJ, 231, 539

Dhawan, V., Mirabel, I. F., \& Rodríguez, L. F. 2000, ApJ, 543, 373

di Salvo, T., Iaria, R., Burderi, L., \& Robba, N. R. 2000, ApJ, 542, 1034

di Salvo, T., Robba, N. R., Iaria, R., et al. 2001, ApJ, 554, 49

di Salvo, T., Stella, L., Robba, N. R., et al. 2000, ApJ, 544, L119

Fender, R. 2003 [arXiv: astro-ph/0303339]

Fender, R. P., \& Hendry, M. A. 2000, MNRAS, 317, 1

Fleischman, J. R. 1985, A\&A, 153, 106

Fomalont, E. B., Geldzahler, B. J., \& Bradshaw, C. F. 2001, ApJ, 553, L27

Galloway, D. K., Psaltis, D., Chakrabarty, D., \& Muno, M. P. 2003, ApJ, 590, 999

Garcia, M. R., Grindlay, J. E., Bailyn, C. D., et al. 1992, AJ, 103, 1325 
Garcia, M. R., Grindlay, J. E., Molnar, L. A., et al. 1988, ApJ, 328, 552

Grindlay, J. E., \& Seaquist, E. R. 1986, ApJ, 310, 172

Gruber, D. E., Blanco, P. R., Heindl, W. A., et al. 1996, A\&AS, 120, C641

Hasinger, G., \& van der Klis, M. 1989, A\&A, 225, 79

Hjellming, R., \& Han, X. 1995, in X-ray binaries, Cambridge Astrophys. Ser., ed. W. H. G. Lewin, J. van Paradijs, \& E. P. J. van den Heuvel (Cambridge, MA: Cambridge University Press), 308

Hjellming, R. M., Han, X. H., Cordova, F. A., \& Hasinger, G. 1990a, A\&A, 235, 147

Hjellming, R. M., Stewart, R. T., White, G. L., et al. 1990b, ApJ, 365, 681

Homan, J., van der Klis, M., Wijnands, R., Vaughan, B., \& Kuulkers, E. 1998, ApJ, 499, L41

Hua, X., \& Titarchuk, L. 1995, ApJ, 449, 188

Iaria, R., Burderi, L., di Salvo, T., La Barbera, A., \& Robba, N. R. 2001, ApJ, 547, 412

in 't Zand, J. J. M., Verbunt, F., Strohmayer, T. E., et al. 1999, A\&A, 345,100

Jahoda, K., Swank, J. H., Giles, A. B., et al. 1996, Proc. SPIE, 2808, 59

Jonker, P. G., van der Klis, M., Homan, J., et al. 2002, MNRAS, 333, 665

Jonker, P. G., van der Klis, M., Wijnands, R., et al. 2000, ApJ, 537, 374

Kellermann, K. I., \& Pauliny-Toth, I. I. K. 1969, ApJ, 155, L71

Klein-Wolt, M., Fender, R. P., Pooley, G. G., et al. 2002, MNRAS, 331,745

Matsuba, E., Dotani, T., Mitsuda, K., et al. 1995, PASJ, 47, 575

Migliari, S., Fender, R., Rupen, M., et al. 2003, MNRAS, 345, L35

Mirabel, I. F., Dhawan, V., Chaty, S., et al. 1998, A\&A, 330, L9
Muno, M. P., Remillard, R. A., \& Chakrabarty, D. 2002, ApJ, 568, L35

Penninx, W. 1989, in 23rd ESLAB Symp. on Two Topics in X-Ray Astronomy, ed. J. Hunt, \& B. Battrick, ESA SP-296, 1, 185

Penninx, W., Lewin, W. H. G., Zijlstra, A. A., Mitsuda, K., \& van Paradijs, J. 1988, Nature, 336, 146

Penninx, W., Zwarthoed, G. A. A., van Paradijs, J., et al. 1993, A\&A, 267, 92

Pooley, G. G., \& Fender, R. P. 1997, MNRAS, 292, 925

Rothschild, R. E., Blanco, P. R., Gruber, D. E., et al. 1998, ApJ, 496, 538

Schnerr, R. S., Reerink, T., van der Klis, M., et al. 2003, A\&A, 406, 221

Schulz, N. S., Hasinger, G., \& Truemper, J. 1989, A\&A, 225, 48

Sidoli, L., Parmar, A. N., Oosterbroek, T., \& Lumb, D. 2002, A\&A, 385,940

Stella, L., White, N. E., \& Taylor, B. G. 1985, in Recent Results on Cataclysmic Variables, 125

Tan, J., Lewin, W. H. G., Hjellming, R. M., et al. 1992, ApJ, 385, 314

Titarchuk, L. 1994, ApJ, 434, 570

Ueda, Y., Asai, K., Yamaoka, K., Dotani, T., \& Inoue, H. 2001, ApJ, 556, L87

van der Klis, M. 1995a, in X-ray binaries, Cambridge Astrophys. Ser., ed. W. H. G. Lewin, J. van Paradijs, \& E. P. J. van den Heuvel (Cambridge, MA: Cambridge University Press), 252

van der Klis, M. 1995b, in Proc. NATO Advanced Study Institute on the Lives of the Neutron Stars, held in Kemer, Turkey, August 29-September 12, 1993, ed. M. A. Alpar, U. Kiziloglu, \& J. van Paradijs (Dordrecht, The Netherlands, Boston, Massachusetts: Kluwer Academic), 301

Wijnands, R. A. D., van der Klis, M., Kuulkers, E., Asai, K., \& Hasinger, G. 1997, A\&A, 323, 399

Zhang, W., Giles, A. B., Jahoda, K., et al. 1993, Proc. SPIE, 2006, 324 